\title{
WILKIE'S DISEASE
}

(A Case Report)

\author{
Col KK MAUDAR*, Lt Col R SOOD ${ }^{+}$, \\ Maj HS BINDRA ${ }^{+}$
}

MJAFI 1997; 53 : 59-60

KEYWORDS : Intestinal obstruction; Superior mesentric artery; Wilkie's disease.

\section{Introduction}

$\mathbf{W}$ ilkie's disease is an unusual form of high intestinal obstruction, and was postulated by Rokitansky in 1842 . The condition has been called by various names and there is no unanimity about terminology. It has been termed as duodenal ileus, gastromesenteric ileus, vascular compression of duodenum, and superior mesenteric artery syndrome [1]. A rare case is being reported.

\section{Case Report}

A tall thin 35-year-old male factory worker was admitted with epigastric pain and repeated vomiting of one week's duration. He had been hospitalized three times in last 2 years with similar complaints and had responded to conservative treatment each time. Clinical examination and plain radiograph of abdomen were non-contributory. He was managed by nasogastric suction and fluid therapy. The symptoms werc relieved within 72 hours. Within a week of relief of symptoms, he was investigated by barium meal studies which showed dilated stomach and minimal gas distally. There was gross dilatation of 2 nd and 3 rd part of the duodenum, vertical linear pressure effects on the 3rd part of duodenum and delayed passage of contrast, suggestive of Wilkie's disease. Exploratory laparotomy confirmed the diagnosis. Side-to-side duodenojejunostomy was done. Post-operative recovery was uneventful. Individual has been followedup for one year and he is asymptomatic and has gained weight.

\section{Discussion}

Due to man's erect posture the superior mesenteric artery grooves the anterior surface of 3rd part of duodenum. The acute aorto-mesenteric angle compresses this part of the duodenum in a nutcracker fashion [2]. Wilkie's disease is common in tall, thin, young adolescents, and there is a slight female predominance [2]. Fat and lymphatics around the origin of superior mesenteric artery protect the duodenum from compression. Cases are rarely seen in obese individuals. There are 2 types of Wilkie's disease, acute and chronic. Acute symptoms sometimes develop after sudden weight loss (as in severe burns, cancer, anorexia, nervosa), post-operative period, spinal trauma and as a part of the cast syndrome [3]. The acute type is less common and presents as upper gastrointestinal obstruction, with repeated vomiting being the predominant feature. Gastric dilatation may occur, leading to an audible succussion splash. In the chronic type, epigastric discomfort and fullness occur after meals, with vomiting providing relief to the pain. Foul eructations are common. Individuals also gives history of loss of weight [4].

Plain radiograph of the abdomen shows a double bubble appearance specially in children. Barium meal study shows duodenal dilatation proximal to the 3rd part and vertical linear extrinsic pressure in the same region, as seen in our patient. There is a delay in passage of contrast material and in the prone position, but there is a quick and complete emptying. These findings may be absent and hence repeated examinations are recommended. Hypotonic duodenography is a provocative test [5]. Angiography is not of much use. Only upper gastrointestinal endoscopy can rule out tumours and duodenal webs as the cause of obstruction [57].

Conservative treatment is preferred and should be tried first, specially in patients with a short history, moderate symptoms, and minor degree of duodenal stasis. Conservative management was followed in the present case also. The patient is to

"Professor \& Head of Surgery, Dept of Surgery, Armed Forces Medical College, Pune 411040; ${ }^{+}$Ctassified Specialist (Surgery), Military Hospital Jabalpur. 
be advised to remain in the prone, left lateral position after meals. Cast syndrome in orthopaedic cases requires modification in treatment approach, so that the individual can be ambulated or use rotating frame bed. If the individual does not respond to adequate conservative treatment, the operation of choice is a side-to-side duodenojejunostomy. This procedure when performed in our patient relieved the recurrent attacks of duodenal obstruction completely.

\section{REFERENCES}

I. John PW. Miscellaneous causes of small bowel obstruction. In: Bowel Obstruction, Differential diagnosis and clinical management. Philadelphia: WB Saunders, 1990;
449-96.

2. Jones SA, Carter R, Smith LL. Anterior mesenteric compression. Am J Surg 1960; 100: 262-77.

3. Wayne E, Miller RE, Eiseman B. Duodenal obstruction by the superior mesenteric artery in bedridden combat casualties. Ann Surg 1971; 174: 339-45.

4. Vohra R, Saini l. Duodenal ileus presenting as acute upper gastrointestinal obstruction. Aust NZ J Surg 1882; 52: 512.

5. Agrawal JB, Swami RS. Wilkie's disease. Indian Joumal of Surgery 1992; 54: 234-5.

6. Vadi RI, Shah GS. Superior mesenteric artery syndrome. Indian Journal of Surgery 1987; 49: 430-3.

7. Singh KP, Gupta M, Sethi MS. Chronic duodenal ileus. Indian Journal Surgery 1983; 45: 553-8. 